\title{
Scaffolding Scientific Conceptualization: \\ Multiple representation and multilevel visualization using an iconic modeling tool
}

\author{
Nancy LAW \\ The University of Hong Kong
}

\begin{abstract}
Difficulties in the teaching and learning of scientific concepts and theories have been well documented in many areas of science, including the physical and life sciences, over the last two decades. Different psychological theories have been put forward as explanatory models for such difficulties. However, another perspective for understanding such learning difficulties and consequently to better support learning is to understand the relationship between the theories and the phenomena they explain. Often, scientific theories involve the postulation of explanatory models that operate at an entirely different level (or scale) in terms of the magnitude and time span from the natural phenomena that it seeks to explain. This paper elaborates the challenges to learning that this poses and introduces how an iconic modeling tool, WorldMaker, can scaffold conceptualization in the learning of a variety of scientific theories.
\end{abstract}

\section{1: Iconic modeling: creating systems of interacting entities}

An iconic modelling tool, WORLDMAKER, was designed as an easy-to-use, intuitive modelling environment built on the basis of an intermaths system similar to cellular automata machines [1] to support reasoning about complex systems that would be accessible even to young children $[2,3]$. In this environment, users can build models through defining entities (objects and backgrounds) and events (interactions between entities in the "world"). In this environment, children are encouraged to think of rules of interactions as possible events that can occur in the "world".

\section{1: The concept of the world in WorldMaker}

The modelling environment in WorldMaker 2000 is based around a "World" which consists both of the map here the foreground and background objects are placed, as well as the rules governing the interactions and actions of these objects. The size of this is defined when a new world is created.

\section{2: The main interface}

The main interface (figure 1) contains all the controls used for the manipulation of worlds and other controls. This screen contains various sets of controls for different purposes for the multi-user version, which supports remote co-construction of models.

The first step in the creation of a model of a system of interacting entities is the definition of the variety of entities to be found in the system.

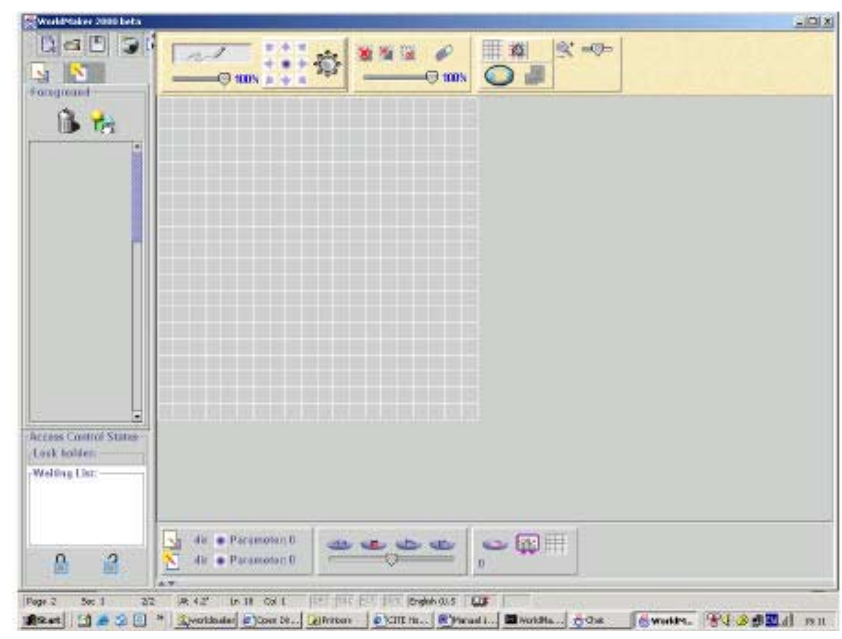

Figure 1. The main user interface for WorldMaker showing the worldgrid and the entities panel for object/background selection

\section{3: The rule definition panels}

Interactions between different types of entities can only take place if they are co-located with each other. Such interactions can be defined through the rule definition panel (see figure $2 \mathrm{a} \& 2 \mathrm{~b}$ ).

Details about the system and how it works can be found at http://worldmaker.cite.hku.hk. Two versions of the tool are available, one running on the Windows environment in standalone mode only, while the other is a java version that supports remote collaborations on the same model. User manuals for both versions can be found 
at

http://worldmaker.cite.hku.hk/worldmaker/pages/manuals. htm.

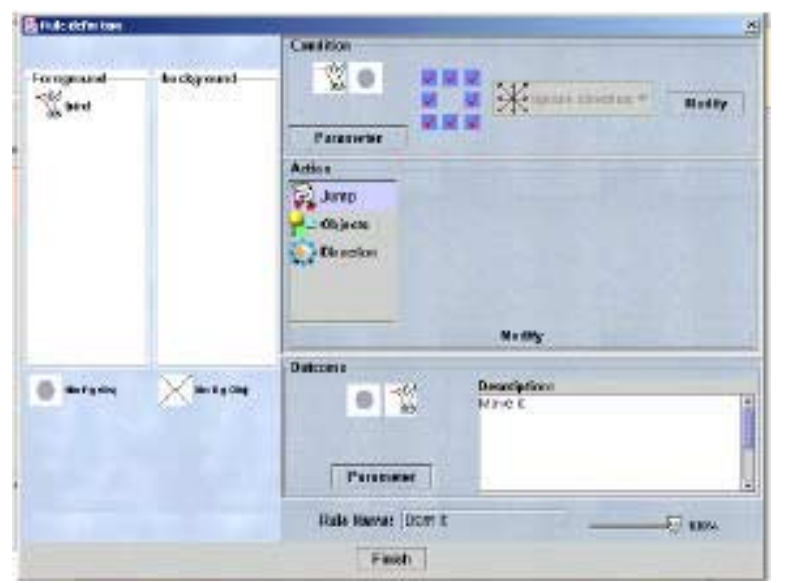

Figure $2 a$ The rule editing panel for defining interactions between an entity and its neighbours.

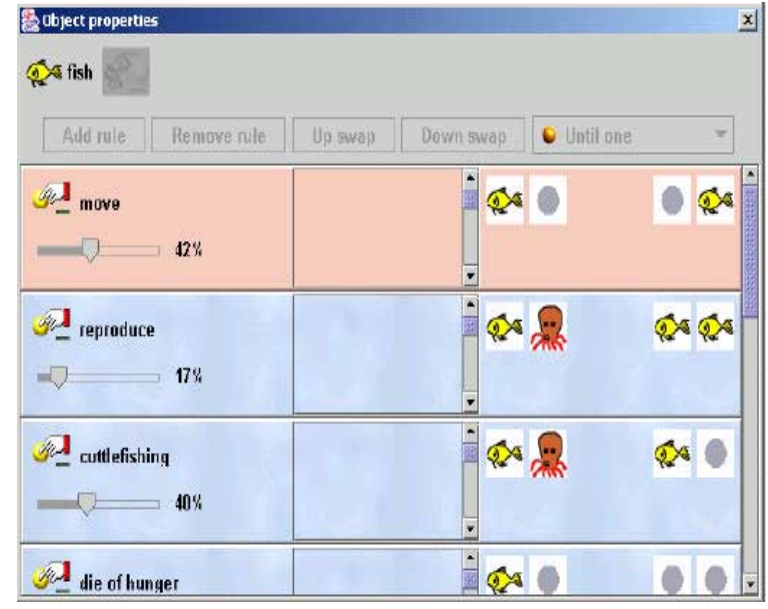

Figure 2b Each entity can interact with its immediate environment through multiple rules.

\section{2: WorldMaker rules: kinds of interactions supported}

WORLDMAKER is different from a cellular automata system in that each cell can contain up to two entities: a 'background' and an 'object'. The latter being able to move while the former cannot. Besides movement, the system can be used to define rules that lead to transformation of entities, or a change in direction of the entities. With these three broad categories of rules, many different kinds of physical or biological interactions/ transformations can be simulated.

\subsection{Movement rules}

There are two kinds of movement rules that can be defined in WorldMaker: jump and roll. Jump does not require the object to possess direction, and an object can roll into a neighboring location if it is not occupied. A simple model of diffusion can be created by defining each entity in the world to be capable of making random jumps. Different diffusion rates can be simulated through defining different jump probabilities. Roll, on the other hand, requires that the object possesses a direction before movement can take place. An object can roll into a neighboring location that it pointing to if that location it is unoccupied. Models of colliding objects can be created by setting objects to continue rolling in a particular direction until it hits an obstacle.

\subsection{Direction rules}

Rules can be defined to change the directions of objects and backgrounds under a variety of conditions. For example, a rolling object can "bounce back" by defining its direction to change by $180^{\circ}$ when it hits an obstacle. Likewise, spinning or oblique collisions can also be defined through setting different changes in direction upon interaction. Field effects can also be simulated by setting direction change rules for an object to pick up the direction of the background it is located on.

\subsection{Entity transformation rules}

This is by far the most interesting set of rules which allows for a large diversity of simulations to be created, especially when used in conjunction with the movement rules. Objects can grow to a next stage of development (e.g. from baby to adult), decay or metamorphose by defining rules that allow an object to change into another. An object may also disappear or die by defining that an object has a specific probability of disappearing. If such transformation requires specific pre-conditions, then this type of object transformation rule can be interpreted as transformed, changed or destroyed by neighbour, such as killing of a prey by a predator located next to it. Rules can also be defined such that new objects can be created. Such rules can be interpreted as reproduction or growth in size, as the case may be. Depending on the pre-conditions required for the rule to fire, the interaction can be interpreted in an even wider diversity of settings, e.g. vapor particles condensing, animals mating so that the female becomes pregnant, etc. 


\section{3: Challenges for understanding scientific theories: issues of visualization and representation}

The advantage of using an iconic modeling tool to creating models of natural phenomena to support the teaching and learning of science is that it offers a unique opportunity for the user to visualize phenomena at two levels: the local, often probabilistic, interaction level and the global, often deterministic behavioral trend level. Scientific theories often involve the postulation of explanatory models that operate at an entirely different level (or scale) in terms of the magnitude and time span from the natural phenomena that it seeks to explain. Depending on the specific subject matter domain involved, the difficulty that learners have in conceptualizing the scientific theory may lie at the microscopic interaction level or the global behavioral trend level. WorldMaker can scaffold learning by helping the learner to visualize how these two levels of understanding the same phenomenon is related.

\section{1: Concrete, "life -size" local interactions and abstract global theories}

One category of difficulty is in conceptualizing global trends which are often non-intuitive for systems where the phenomenon at the local interaction level is very familiar and well understood. An example of this kind of difficulty in science is in understanding the environmental impact of introducing different influences to the ecological environment. Predator-prey relationship at the local interaction level is very well understood even by young children: foxes eat squirrels and squirrels eat nuts; both red and black squirrels eat nuts. Why is the introduction of black squirrels mo re threatening to red squirrels than the introduction of foxes to an indigenous environment where there was only red squirrels, no black squirrels and no foxes. Conceptualizing the introduction of a "harmless" competitor to be even more environmentally destructive than the introduction of predator has been found to be difficult even for adults, including teachers. However, when young children were given the opportunity to explore ecological impacts of various environmental changes, they can provide explanations for this and other related ecological principles such as why non-indigenous species, not even plants, should be introduced to an indigenous ecology, and why some species, especially those at the top of the food chain, may be endangered by increasing urbanization.

The nature of the challenge in conceptualizing ecological trends and principles is that human beings are participating in the ecologic system at a microscopic (or local) interaction level both in terms of size (constraining us to not be able to see the overall impact as an aggregate of effects over wide geographic spans) and time span (ecological impacts takes generations (in terms of the life spans of the affected species, which than becomes difficult to observe over our own human life span). Using WorldMaker, the user can visualize the interaction at the local interaction level (squirrel eaten by fox, or squirrel die of hunger) as well as see how the total count of squirrels at any one time. Further, generations of squirrels can elapse within seconds of running the simulation.

\section{2: Concrete, deterministic global observations and abstract microscopic theories}

Another class of difficulty involves difficulties in conceptualizing the microscopic interactions. One prime example of such is the difficulty involved in understanding the myriads of physical phenomena associated with the particulate theory of matter. We are all familiar with the gas laws and related phenomena (e.g. gas pressure increases when heated under constant volume, gas cylinders explode when placed near heat sources), as well as many diffusion related phenomena such as spreading of fragrance, Brownian motion and natural mixing of soluble liquids. However, to relate these macroscopic observations to the microscopic theory of the particulate theory of matter and the assumptions of the kinetic theory of gases is not intuitive at all. In fact it is not obvious why one would wish to look for an explanatory model for such macroscopic observations at this very abstract, apparently unrelated, microscopic level. Why should scientists be looking for explanatory models of macroscopic phenomena from a microscopic theory of matter are too complex to be dealt with in this paper. However, to even understand and appreciate that the physical phenomena as a necessary outcome of the microscopic theories per se is already very complex and very challenging even for senior high schools. It was found that getting students to construct their own models to explain for the variety of observable phenomena at least allow them to see the plausibility and the connections between the two levels of understanding of the same phenomena. Some also started to appreciate that one of the beauty of the particulate theory of matter is the simplicity and ubiquity of the theory for explaining widely diverse phenomena.

\section{3: Understanding probabilistic rules}

Another area of difficulty that students often have in understanding is the meaning of the probability in probabilistic rules. In fact, it is generally not easy for students to fully grasp the meaning of predictability (in the sense of having predictable probabilities) of random events. 
The simplest example of this category of difficulty is that of radioactive decay. A mass consisting of an aggregate of component particles, type A, each of which have the same probability of decaying into another type of particle, type $\mathrm{B}$, will be having a changing composition as time goes on. The quantity of type A particles will decrease with time, not linearly but exponentially. Students find it very difficult to understand the decay phenomenon as having a deterministic trend and yet there is no way to predict how long a specific particle may take for the decay to take place [3]. However, observing the radioactive decay simulation in WorldMaker, students were able to see how particles randomly undergo decay on the simulation screen and that given a finite number of radioactive particles, all would eventually undergo decay, though the exact time taken would be unpredictable. This helped students to gain a better understanding of the concepts of decay probability and half-life in radioactivity.

Genetic models of heredity and propagation of traits are more difficult theories within this same category. According to Longden [4], students generally understood the genetics ratio as a fixed number and routinely used the punnett squares to solve genetics problems. Following this, Kinnear [5] pointed out that students perceived genetics ratios as a deterministic parameter rather than a probabilistic one. This inability to understand the probabilistic nature of genetic phenomena is one major obstacle to the learning of genetics concepts.

Here, WorldMaker simulations can also provide a helpful visualization tool to support the development of understanding. In a field trial conducted with a group of senior secondary science students, an exploration was conducted on the distribution of coat colour for offspring from different parental genotypic combinations. They were examining the offspring from 2 heterogeneous parents:

"Oh! Why the ratio are different at time period 10, 20 and so on?"

"Yes, it is not equal to 3:1. That is strange."

"Let us calculate the exact ratio and correct to 1 decimal place. 11:4 is equal to 2.8 and 19:6 is equal to 3.2, 33:10 is equal to 3.3. and 48:15 is 3.2. It is not exactly equal to $3: 1$. ."

"Um. Why? Maybe... Ah! I remember it is random. 3:1 is only an ideal ratio."

"Yes, it is just like tossing a coin, 50\% of having a tail is just an ideal case.

Through the simulation, students soon realized the probabilistic nature of the genetic ratio.

\section{4: Multiple visual representations of trait (genetic) theories}

A third category of scientific theories that are difficult to understand does not arise from two levels (or scales) of experiencing/visualizing the same phenomena, but in visualizing (and thus predicting) the (deterministically) probabilistic outcomes arising from interactions of known probabilities. Another major source of difficulty in visualizing the propagation of genetic traits relates to the fact that often multiple genetic compositions can be mapped to the same observable traits because of the existence of dominant and recessive genes. Using the technical terminology in genetics, this difficult relates to the fact that there are multiple genotypes associated with the same phenotype (a phenotype is a group that shares the same observably identifiable characteristics while genotype refers to a group that shares not only the same physical characteristic, but also the same genetic composition).

In WorldMaker, the graphic image of the icon used to represent an entity is totally independent of the rules that defines the entity's behavior. Thus one can easily create different versions of the same simulation with different graphic representations to aid learners in their understanding. For example, Figure 3 shows two sets of icons that can be used to represent the three rabbit genotypes. For the phenotype "white rabbit", there is only one genotype, " $\mathrm{tt}$ ". However, for the phenotype "brown rabbit", a particular brown rabbit can be either of two possible genotypes, "Tt" or "TT", the former comprising of one dominant and one recessive gene while the latter comprises two dominant genes. Thus when two brown rabbits mate, or one brown rabbit and one white rabbit mate, the result can be very different depending on the genotype of the brown rabbit involved. As WorldMaker supports easy editing of the iconic representation for entities, two simulations that shares exactly the same set of rules of genetic inheritance rules for the same genetic entities but with different iconic representations can be created. It was found that by moving between these two different iconic representation of the same entity, students can understand not only the subtle difference between phenotype and genotype, but be able to predict the likely offspring composition for different parental traits.

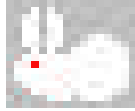

A

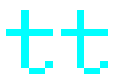

E

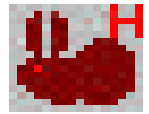

B

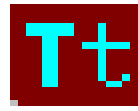

F

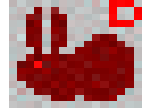

C

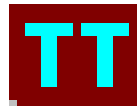

G
Figure 3. Two sets of icons for representing the three genotypes of rabbits with 2 colour coats.

In one field trial, a group of grade 12 students used a simulation created in WorldMaker (using icons a,b \& c) 
to explore the breeding behavior of rabbits. They were surprised to find white rabbits being born when two brown rabbits mated. Students raised the following questions:

"Is it due to the fact that the parent rabbit gave birth to brown rabbit first then pass on the white one?"

"Do you mean that the parent rabbits are running out of pigments so the colour will become paler and paler?

"Does the quality of the grass [that the rabbit eats] affect the result?"

Students thus proposed a variety of conjectures to try and explain why a white rabbit can be born from brown parents. Then some of the students took notice of the red letters marked on the icon of the brown rabbits and asked:

"What does the red letters represent? Will these affect the whole system?"

"Two [brown] rabbits look the same in appearance but have different letters marked, does this matter?"

Here, in this conversation, the "letters" referred to the letters " $D$ " \& " $H$ " marked on the brown rabbit icons shown in Figure 3. One great feature of WorldMaker is that it allows students to formulate and test hypothesis and reconcile any discrepancy between their ideas and observation. For example, the above group of students was later introduced to the same simulation created using icons $\mathrm{d}$, e \& $\mathrm{f}$. They were able to discuss and formulate further hypothesis and learn from the feedback from the simulation:

"Oh! So here are two brown rabbits, two pure breed brown rabbit."

"What I have interpreted is that if you have two of these (pure bred dominant), the offspring will always be brown." (Drawing the Punnett square illustrating his thought.)

"So this is the case for two pure bred brown rabbits. What if there is a brown rabbit with $T$, and with another pure bred white rabbit with. What will be the colour of their offspring?"

"So there are 4 possibilities: Tt, Tt, tt and tt, half of the offspring will be white and half will be brown."

"Oh! But why isn't it similar to the present situation?"

\section{4: Iconic modeling for scaffolding scientific conceptualization}

In summary, this paper illustrates that much of the difficulties in conceptualizing (which is the first step towards understanding) scientific theories relates to the fact that scientific theories involve the creation of abstract theoretical constructs which are not only not visually observable, but that there are often intrinsic difficulties for the learner to relate these constructs to the phenomena studied. The iconic modeling tool WorldMaker provides a simple tool for the user to create models involving locally interacting entities (foreground and background objects) and supports visualization of the same phenomenon from both the microscopic interactional perspective and the global behavioral trend perspective. Such a tool can be used to scaffold learning in science for three categories of difficulties were identified.

Some phenomena like predator-prey relationships are easily accessible and understood at the local interactional perspective but understanding the global ecological impacts of environmental changes are difficult because it involves visualizing the same phenomenon from another level involving a much wider geographical scope as well as much longer time span for observation compared to our everyday experience. On the other hand, some phenomena are easily observable at the global behavioral level, such as gases laws, phase transitions and diffusion phenomena. However, to appreciate the particulate model of matter and that such a model can be used to explain ubiquitously this whole range of behavior is much more difficult because the theoretical constructs created are abstract and the kinds of interactions proposed in the model are not generally observable in our everyday life. A third category of difficulty relate to trait (genetic) theories. Here, two main difficulties are involved. The first one is the difficulties involved in visualizing probabilistic interactions and relating these to deterministic behavioral outcomes at a global scale. The second type of difficulty relates to multiple versions of one abstract theoretical construct (genotype) being associated with one single version of a more concrete, observable construct (phenotype). It has been found that WorldMaker provides a very fruitful tool to scaffold the learning of many different scientific theories belonging to these three categories by supporting multilevel visualization of the same phenomena and multiple representation of the same entities.

\section{References:}

[1] Toffoli, T. a. M., N. (1987). Cellular Automata Machines: A New Environment for Modelling. Cambridge, Massachusetts: MIT Press.

[2] Law, N. \& Tam, E. (1998). WORLDMAKER (HK)- an Iconic Modelling Tool for Children to Explore Complex Behaviour.Paper presented at the International Conference on Computers in Education. Chiba.

[3] Law, N. (1999). Making Physics Concepts Accessible and Explorable Using WorldMaker - an iconic modelling tool. Paper presented at the International Conference on Physics Teaching. Guilin, PRC. August 17-21, 1999.

[4] Kinnear, J. (1983). Identification of misconceptions in genetics and the use of computer simulations in their correction. Paper presented at the International Seminar on Misconceptions in Science and Mathematics.

[5] Longden, B. (1982). Genetics - are there inherent learning difficulties. Journal of Biological Education 16(2) 135-140. 